\title{
GRANDES URBES \\ Y NUEVAS VISIBILIDADES \\ DE LA DIVERSIDAD
}

\author{
Liliana Ester Tamagno \\ Carolina Andrea Maidana
}

1 Algunas cuestiones que aparecen en este trabajo fueron presentadas y debatidas en el VIII Congreso Latinoamericano de Sociología Rural, Porto de Galinhas, Brasil, 2010.

R E S U M E N En el presente trabajo reflexionaremos sobre las dialécticas: tradición/ modernidad, locallglobal, formallinformal, pasado/presente, memoriaslaprendizajes en su relación con las migraciones indígenas, y las respuestas de esta población a las condiciones de la vida urbana. El análisis de las migraciones internas entendidas como desplazamientos de individuos y/o colectivos en busca de trabajo y de mejores condiciones de existencia (Castells, 1979 y Tamagno, 2001) y la relación entre urbanización, desigualdad y diversidad se realiza en este trabajo poniendo énfasis en los denominados "procesos de territorialización", noción que nos permite dar lugar a la comprensión de las formas en que los territorios de pertenencia se construyen históricamente como resultado de la conjunción de factores politicos, económicos y socioculturales; algo de suma importancia dado el actual contexto de demandas territoriales y de la consecuente lucha por legitimar las presencias indígenas en el medio urbano. ${ }^{1}$

P A L A B R A S C L A V E Ciudad; indígenas; migración; nucleamientos; procesos de territorialización.

\section{AMÉRICA LATINA: PROCESOS MIGRATORIOS Y URBANIZACIÓN}

En América Latina, en la década de 1960, el tema de las migraciones internas fue preocupación de científicos sociales, economistas, agentes sociales y políticos, y debido a la magnitud de las mismas, produjo un desmesurado aumento de la población de las ciudades en períodos muy cortos de tiempo, derivando situaciones preocupantes. Las denominadas entonces, "migraciones internas", referían a los sectores más pobres de la población del interior de los países que, masivamente, se dirigían a las ciudades en busca de mejores condiciones de existencia. Finalizada la Segunda Guerra Mundial, la inversión abrupta de capitales -ahora ociosos- en áreas periféricas dio lugar a una etapa de significativo desarrollo industrial, acompañada por un desplazamiento de inversiones de capital del campo a la ciudad que provocó estos movimientos de población los cuales fueron caracterizados como "oleadas migratorias", "aluviones poblacionales" o "aluviones zoológicos", por quienes se atrevieron a expresarlo en términos claramente conservadores y racistas. En Argentina esta última frase, atribuida al diputado radical Ernesto Sanmartino en un debate de la Cámara de Diputados del 07/08/1947 (Gambini, 2008), pasó a formar parte del imaginario colectivo asociada a la descripción de sectores populares -mayoritariamente peronistas- conjuntamente con los calificativos "negros", "negritos", "negrada", "grasas".

El campo se despobló y las ciudades crecieron desmesuradamente al ritmo de la industrialización, de la especulación inmobiliaria y de la revaloración de la tierra urbana. Aparecieron así, en América Latina, las llamadas "hiperurbes" y sus "conurbanos" como 
2 Este momento - en que los gobiernos populares fueron combatidos y sofocados - se expresó en Argentina con el Golpe Militar de 1955, lo que significaría un punto de inflexión para pasar de un modelo de redistribución acorde a lo que se denomina "Estado de Bienestar" a un modelo fundado en la libertad de mercado y la acumulación de capital.

3 Para ello fue necesario, en 1954, el Golpe Militar al Gobierno Popular de Getulio Vargas que mostró paralelismos con el Gobierno Peronista en Argentina iniciado en 1946.

4 Buenos Aires, ciudad portuaria, se conecta con el interior del país a través de una red ferroviaria que se semeja a un abanico que priorita las relaciones con el centro. San Pablo es referenciada como "la tercera ciudad de industrial sueca" y se visualiza metafóricamente como una locomotora tal cual lo plantea la obra de Joseph Love (1982). producto de un desarrollo que fue caracterizado como "capitalismo dependiente" (Cardoso y Faletto, 1970), ya que, guiado por las necesidades de expansión y reproducción del capital extranjero y en connivencia con las ansias de rápido enriquecimiento de sus socios locales, obviaba toda posibilidad de planificación en términos de proyectos de países equilibrados y equitativos. ${ }^{2}$ Este crecimiento vertiginoso y desigual hizo que el Conurbano Bonaerense (área integrada por partidos que rodean Buenos Aires, ciudad capital de la Argentina) pasara a contar con 3.700 .000 habitantes en 1960 a 6.800 .000 en 1980. Sólo como otro ejemplo de ello, la ciudad de San Pablo (Brasil) triplicó su población entre los años 1950 y 1970. El Número 7 de la Revista Rapport del Styrelsen För Internationell Utvecklingen (SIDA), Suecia, 1978 - siguiendo el pensamiento socialdemócrata que en ese momento cuestionaba fuertemente el modelo liberal - bajo el título Svensk Indutri i Brasilien (La industria sueca en Brasil) pone al descubierto el modo en que los capitales extranjeros provocaron lo que se denominó "el milagro brasileño"; señalando la significativa participación de Suecia en ese proceso y las enormes ganancias obtenidas durante el mismo. ${ }^{3}$ Más del 50\% del crecimiento de las hiperurbes latinoamericanas se debió entonces, a la migración interna y si bien ésta no se restringió sólo a los sectores más desfavorecidos, fueron éstos los que se vieron enfrentados masivamente a la necesidad de migrar abandonando sus lugares de origen.

La expansión de la economía de mercado trasformó y/o destruyó en el interior de los países, las redes de producción y comercialización locales, la renta se concentró en pocas manos y la economía de los Estados se manejó desde las metrópolis. ${ }^{4}$ Expresando un claro sentido evolucionista, los países latinoamericanos fueron categorizados como "subdesarrollados" y llamados eufemísticamente "pobres" cuando, en rigor de verdad, fueron y siguen siendo, la fuente de riqueza de los países "desarrollados" o "industrializados". Más tarde, y siempre dentro del mismo paradigma, aparecería el término "países en vías de desarrollo" o "países emergentes" para denominar a aquellos que presentaban algún indicador que los mostrase como acercándose al modelo hegemónico. En la década de 1990 se llegó, incluso, a acuñar el término de "países inviables" para designar a aquellos que presentaban balanzas comerciales deficitarias o endeudamiento significativo con pocas probabilidades de hacerse cargo de los mismos y "caer en el default". El desarrollismo en tal paradigma conceptual, pretendió y, aún pretende, ocultar que el "desarrollo" de algunas áreas se realizó y se realiza a costa del "subdesarrollo" de otras, y que los procesos de urbanización presentados como el símbolo de la modernidad, derivarían en importantes desequilibrios y desigualdades regionales que afectaron a toda la sociedad. El paradigma de la "modernización" proveía el fundamento ideológico de que el campo era el atraso y la ciudad el futuro prometedor (Tamagno, 2001). Los que llegaron a la gran ciudad y no pudieron acceder a un trabajo que les garantizara una vivienda digna, pasaron a formar parte de los denominados asentamientos informales. Asentamientos estos caracterizados también como ilegales y/o irregulares, respecto a aspectos dominiales tales como: falta de títulos de propiedad o contratos de alquiler, y/o respecto al incumplimiento de las normativas de urbanización como normas de subdivisión, uso, ocupación, y construcción de la ciudad y/o de los requerimientos ambientales para la localización de usos urbanos (Clichevsky, 2009). Las "villas miserias", "villas de emergencia" o simplemente "villas" de Argentina, las "favelas" de Brasil, las "callampas" de Chile, los "cantegriles" de Uruguay y las "barriadas" de Perú - por nombrar sólo algunas de las expresiones que identifican a este tipo de asentamientos en el espacio latinoamericano - fueron entendidos en un comienzo como signo de progreso, en tanto transitorios. Algunas ciudades comenzaron a 
perder su acentuado tinte europeo y se volvieron multicolores. Buenos Aires dejó de ser la "ciudad europea y blanca" para ir tornándose más "nacional" al recibir, en su seno, a los hijos del interior, a los que el prejuicio y la subestimación denominarían "cabecita negra" y que luego se convertirían en "villeros", o más despectivamente, en "negros villeros". Era sumado así a la diversidad representada en la inmigración extranjera que caracterizó los finales del siglo XXI, la diversidad propia del "interior" representada por los sectores campesino-indígenas.

El neoliberalismo dio continuidad a este paradigma y se impuso decididamente durante las décadas de 1980 y 1990, luego que las dictaduras cívico-militares establecidas en la década de 1970 lograran - a través del terrorismo de estado - acallar las luchas populares que avisoraban la necesidad de avanzar en términos de sociedades más justas y más igualitarias. Nuevos momentos de acumulación de capital complementaron la acumulación originaria que, a partir de la expansión colonial, posibilitó el desarrollo del capitalismo a escala mundial. Altos grados de flexibilidad laboral y desocupación, criminalización de la pobreza y penetración de la droga (Wacquant, 2004) son las consecuencias del despojo que los gobiernos democráticos de hoy observan con preocupación y tienden a pensar en conjunto. Es por ello que se hace evidente que el análisis y tratamiento de las situaciones particulares de cada país latinoamericano debe ser pensado no sólo en el concierto de cuestiones estructurales comunes gestadas en la expansión colonial y proyectadas a lo que se denomina colonialidad, sino en una coyuntura común que implica el hecho de verse obligado a enfrentar, en la actualidad, otro momento de acumulación de capital, representado esta vez por los intereses de los megaemprendimientos sojeros, turísticos, energéticos y por la mega minería. Emprendimientos que implican, a su vez, inversiones de capital en el interior que había sido abandonado durante el comienzo de la segunda década del siglo pasado.

Actualmente, al análisis del fenómeno urbano y los procesos de migración vinculados a la industrialización en tanto agente económico dinámico y en el desarrollo de las ciudades, se suma el análisis de los circuitos financieros y las tramas comunicacionales (Castells, 1996 y García Canclini, 1997) que modifican enormemente no sólo las geografías rurales y metropolitanas, sino también, las experiencias cotidianas del habitar, tanto en el campo como en la ciudad. Las ciudades y la urbanidad aparecen nuevamente comprendidas como expresiones de la relación local-global y de las distintas formas de apropiarse, usar y significar el espacio, desarrolladas en un contexto histórico, político, económico y sociocultural particular.

Giménez (1996) señala que el territorio puede ser apropiado subjetivamente como objeto de representación y de apego afectivo y, sobre todo, como símbolo de pertenencia socio territorial; por ello se puede abandonar físicamente un territorio sin perder la referencia simbólica y subjetiva al mismo a través de la comunicación a la distancia, la memoria, el recuerdo y/o la nostalgia. Son estos mecanismos los que posibilitan la resemantización de los espacios a los que se migra y que pasan a formar parte del territorio rememorado y referenciado. Es por ello que para superar el análisis dicotómico realizado en términos de "desterritorialización"/" reterritorialización" proponemos, la utilización del término "procesos de territorialización" (Oliveira, 1999 y Almeida, 2009) que permite considerar y valorar los aspectos materiales y simbólicos presentes tanto en los destierros como en la ocupación de nuevos espacios (Maidana y Tamagno 2010).

Utilizamos el término nucleamiento ${ }^{5}$ para referirnos a los procesos por los cuales los migrantes indígenas toba (qom) ${ }^{6}$ se reúnen en el espacio y también para describir las áreas de concentración de este tipo de población, es decir, las formaciones espaciales que resultan en "juntarse" en algún espacio particular cuando esto es posible. Entidades territoriales
5 Conceptualización acuñada por Liliana Tamagno (2001) para superar las expectativas de homogeneidad $\mathrm{y} / \mathrm{o}$ de conjuntos cerrados en si mismos.

6 "Qom" (gente) es la forma en la que se autodenominan los integrantes del pueblo indígena conocida como "Toba", pueblo de cazadores, pescadores y recolectores que habitaba, al momento de la llegada de los españoles, la región de América del Sur conocida como Gran Chaco . 
7 Para el caso toba, el nucleamiento más antiguo de Buenos Aires es el de Villa IAPI, municipio de Quilmes. Distrito donde, a mediados del siglo XX, se instalaron grandes empresas debido, tanto a los mejoramientos de infraestructura como a las franquicias impositivas destinadas a fortalecer la radicación de industrias.

8 Para profundizar estas cuestiones ver Tamagno (1986 y 2001). cuyos procesos de gestación, desarrollo y organización son irreductibles a un modelo único, más allá de las generalidades que puedan plantearse al respecto. Algunos nucleamientos están constituidos por conjuntos de familias qom diseminadas en un "barrio", entendido éste como una porción de ciudad definida desde el punto de vista administrativo. Otros nucleamientos están formados por un conjunto de hogares de familias qom, diferenciado entre una mayoría de "criollos" o "blancos". En tanto, otros están constituidos por familias qom nucleadas - en sentido literal - dando lugar a los denominados "barrios tobas", reconociendo a éstos como porciones de ciudad caracterizadas como lugares de cohesión/identificación de los conjuntos de familias que los habitan. Al mismo tiempo, dichos nucleamientos deben ser interpretados como expresión de diferentes modalidades habitacionales resultantes de una ocupación espacial vinculada a la demanda de vivienda que genera, a su vez, el crecimiento - tanto en magnitud como en tipos - de la denominada "informalidad urbana". Incluso algunos casos particulares que en cuanto "viviendas sociales", promovidas por el gobierno nacional, provincial y/o municipal, no serían, en principio, asentamientos informales, ya que se encuentran en situación de "informalidad" cuando el proceso de acceso al suelo no logra formalizarse desde el punto de vista jurídico o bien cuando - debido al crecimiento vegetativo y/o demográfico - se modifican las características de ocupación iniciales (Maidana y Tamagno, 2010).

Siguiendo a Bourdieu (2000), pensamos estos lugares/sitios del espacio social reificado, físicamente realizado u objetivado como apuestas de luchas; como repuestas/resistencias que los propios indígenas dieron y dan ante los procesos de destierro generados por la lógica de la ganancia y el capital, tanto en el campo con el avance de la frontera agrícola y los agronegocios, como en la ciudad debido al avance de la privatización de la tierra y de los negocios inmobiliarios (Tamagno op. cit., y Maidana y Tamagno, 2009). Son numerosos los trabajos que tratan la migración interna en relación a las necesidades de mano de obra, algo que se evidencia en la ubicación de las familias migrantes vinculadas a la proximidad de las fuentes de trabajo. ${ }^{7}$ Sin embargo, para las nuevas generaciones, y dado el aumento de los índices de desocupación, la proximidad a una fuente de trabajo no tiene tanta significación como condicionante de la dimensión territorial.

\section{PUEBLOS INDÍGENAS. LA DIVERSIDAD EN LA CIUDAD}

El acompañamiento de los avatares de un conjunto de familias indígenas qom que migraron al conurbano bonaerense en la década de 1960, permitió reflexionar sobre las falencias de interpretar lo urbano como una dimensión esencializada, como un modo de vida sustancialmente diferente a lo rural, para entender lo "rural" y lo "urbano" como construidos en la dinámica sociocultural de la sociedad que los contiene y de la cual son producto. Planteamos entonces, la necesidad de discutir los límites de la llamada "antropología urbana” (Hannerz, 1980) y tomamos a Manuel Castells (1976) para revisar el "mito de la cultura urbana" e interpretar la ciudad como el lugar donde se concentran y expresan las contradicciones, los conflictos y las desigualdades de la sociedad de la cual la ciudad forma parte. ${ }^{8}$

Hoy, la diversidad resignificada vuelve a aparecer como un desafío para las ciencias sociales, y la metáfora de Maurice Godelier (1978) se torna vigente al permitirnos pensar en términos de un nuevo "parto de la antropología". Planteamos así que los denomina- 
dos sectores populares no pueden ser ya más pensados sin tener en cuenta la variable de la colonialidad y la variable de la etnicidad,lo cual no implica pensar en términos de la necesidad de que los sectores populares se reconozcan en su pasado campesino-indígena, sino en reconocer los procesos sociales que dieron origen a la configuración de los mismos como parte de los Estados nacionales. Los macro fenómenos deben ser así comprendidos atendiendo a las propias interpretaciones y realidades de los conjuntos sociales, en la necesidad de encontrar un vocabulario y unos conceptos que nos permitan integrar la discusión sobre símbolos y significados con las discusiones sobre el trabajo y el mercado, sobre la dinámica de la política, la demografía ${ }^{9}$ y la ecología.

Siguiendo a Barth (1989) es posible analizar la conformación de la complejidad social en términos de corrientes o flujos que tienen una dimensión histórica, donde las circunstancias han hecho que fluyan juntas. Así, las diversidades presentes en lo que denominamos pluralidad no se reducen a la idea de una cultura homogénea, lo que permite comprender el trabajo creativo que los individuos pertenecientes a los grupos étnicos están realizando en el contexto de un mundo en constantes cambios. En el mismo sentido, y como señala Hannerz (1997), no es homogénea en su interior la cultura dominante y tampoco lo son en su interior las culturas subalternas. Participamos en universos de múltiples discursos más o menos discrepantes entre sí; construimos mundos diferentes, parciales y simultáneos y, en ellos, nos movemos y es por ello que la construcción cultural de la sociedad no ocurre y brota de una sola fuente, y no puede ni debe ser pensada como conformando una sola pieza. Según este autor, el concepto de flujo es uno de los términos clave de la llamada antropología transnacional por hacer referencia a aquello que no permanece en su lugar, a movilidades y expansiones variadas. Al mismo tiempo, reconoce a Kroeber como quien no temió pensar la cultura en gran escala y pudo afirmar que las civilizaciones deberían examinarse, no como objetos estáticos, sino como procesos limitados de flujo en el tiempo. Señala a su vez que la noción de flujo puede ser usada de dos maneras: una referida al desplazamiento de algo en el tiempo y a una distribución territorial y otra, específicamente temporal, sin la necesidad de implicancias espaciales. Los flujos tienen direcciones que implican una reorganización de la cultura en el espacio y, si bien existen contraflujos, no pueden negarse las redes de asimetría, vinculadas a relaciones de poder. Las culturas - nos dice - no son visiblemente tan "limitadas", "puras", "homogéneas" y "atemporales" como la tradición antropológica muchas veces las retrata; situación que el mismo Hannerz (1996) analiza en términos de procesos de mestizaje, cuyo carácter puede ser incluso subversivo, en el sentido de cuestionar los significados en circulación.

La ciudad - y esto debe proyectarse a la comprensión de las ciudades latinoamericanas - se nos aparece como un espacio multidimensional. La ciudad no es el "focus" de la denominada cultura urbana, sino el "locus" en el que se desarrollan las culturas de las poblaciones que la habitan, mezclándose y transformándose, compartiendo un sinnúmero de situaciones y, sin embargo, no perdiendo su carácter distintivo, semejando en su efecto, a los flujos o las corrientes de un río. Ello posibilita comprender que las transformaciones que acusan y muestran los indígenas tobas migrantes no implican necesariamente que dejen de ser indígenas, pues el límite o frontera que los separa de los demás permanece toda vez que mantienen su distintividad y desde ella se relacionan con el contexto. Precisamente porque se transforman, ya que las apelaciones al pasado presentes en sus prácticas y sus representaciones en el medio urbano no deben interpretarse como meras expresiones del pasado o de la tradición y, menos aún, como indicadores de atraso. Las apelaciones al pasado son realizadas desde el presente y proyectadas al futuro. Tanto el pasado, como el
9 La importancia de complementar los análisis antropológicos con los análisis demográficos fue enfatizada por François Lartigue en la conferencia que dictara en el Seminario Internacional "Indígenas en el Siglo XXI" en el año 1998, en la ciudad de Buenos Aires, Argentina. 
presente y el futuro son tratados en el marco de las relaciones interétnicas y en el contexto de la sociedad nacional de la que forman parte, aunque ésta, racista y desigual, no les permita gozar de los bienes y los servicios que gozan los sectores más privilegiados (Barabas, 1989 y Bartolomé, 1997).

Si bien el análisis de la situación de las familias qom migrantes a las grandes ciudades como Buenos Aires, Rosario y La Plata implicó en un primer momento analizar la ciudad, la ruptura con la dicotomía rural/urbano y los saberes y demandas de estas familias nos "fueron llevando a los lugares de origen". Observamos los modos en que el Chaco, de donde provienen, está presente en el cotidiano de los barrios periféricos que habitan expresado tanto en representaciones como en prácticas. Las respuestas que los tobas dan a las condiciones que la vida urbana implican una dialéctica entre la "tradición" y la "modernidad" entre lo "local" y lo "global", entre lo "formal" y lo "informal", entre el presente y el pasado, entre la memoria y los aprendizajes. Así, la presencia de indígenas en las ciudades deja de aparecer como una contradicción o como una paradoja, pasando a contribuyer a una ruptura con el modelo de ciudad europea pensada y diseñada bajo los cánones de la modernidad. La "invisibilidad" que otrora sufrieran los pueblos indígenas se transforma en emergencia y la "entelequia" de país venido de los barcos que caracterizó el proyecto de la Generación de 1880 se desvanece para hacernos repensar, no sólo la ciudad, sino la sociedad de la que la misma forma parte, sociedad que no puede dejar de asumir su diversidad.

Hoy una tercera generación de jóvenes que ya no son migrantes, expresan, en el cotidiano de su existencia, esta dialéctica; valorando los saberes de sus mayores se suman -incluso sin proponérselo- a la reproducción de modos alternos de pensar la vida y de pensar y habitar los espacios que implican un serio cuestionamiento al nuevo momento de acumulación de capital. Los versos siguientes corresponden a un tema musical de los realizados por un grupo de jóvenes qom y pueden ser una expresión de lo antedicho:

(...) Contando el pasado pesado de nuestra historia,

Que nunca fue pisado ni olvidado,

La historia que nos sigue marcando,

Como el centro de este marco,

Donde estamos presentes,

Pero para muchos ausentes,

Y seguimos luchando por nuestra cultura,

Desatándonos de estas ataduras,

Porque muchos no quieren ver brillar al hombre originario,

Hombre qom que camina en este barrio $(. . .)^{10}$

10 El tema musical forma parte de un $C D$ producto del Proyecto de Capacitación en Tecnologías de la Información y Comunicación para Jóvenes Toba (Qom), @ 2009.

\section{CIUDAD Y PROCESOS DE TERRITORIALIZACIÓN}

Explicar la presencia de indígenas en la ciudad como el producto de las transformaciones de la sociedad de la cual forman parte, permite comprender que su situación no deriva del aislamiento, sino de los modos en que están insertos en un orden social injusto, construido en el marco de relaciones capitalistas de producción; como también pone en discusión conceptos como desarrollo, marginalidad y exclusión: nunca estuvieron, ni están, a los márgenes y, tampoco estuvieron o están excluidos. Los indígenas que lograron 
sobrevivir al aniquilamiento por guerra o por enfermedades, por arrinconamiento o explotación, no siguieron indefectiblemente el camino de la aculturación y la destribalización que tan enfáticamente pronosticara el desarrollismo y que las trompetas de la "globalización" hacen resonar nuevamente. Lo comunitario y las identidades étnicas persisten,sin embargo, etnicidad no implica homogeneidad. Hay muchas formas de ser indígena, hay muchas formas de ser qom. La identidad étnica se expresa en el hecho de reconocerse en un origen y en una historia común y en actuar de modo comunitario, de pensar y de actuar, sabiendo que pertenecen a un conjunto mayor. La etnicidad se funda, además, en relaciones de parentesco, siendo éste un valor que permite la reproducción del grupo y sus intereses. La identidad se refuerza también en el mantenimiento de la lengua y en la conformación de una comunidad de habla (Ibañez Caselli, 1995 y Ibañez Caselli y Tamagno, 1999). La identidad religiosa no es excluyente de la identidad étnica, toda vez que el ritual canaliza y expresa lo comunitario y toda vez que da fuerza y sentido de cuerpo. La identidad aún es política en el sentido de que coadyuva a la sobrevivencia en un medio signado por la dominación y la desigualdad y en la medida en que expresa los intereses del grupo. La identidad étnica tampoco es excluyente de una identidad de clase, es indígena y ciudadano pobre de un país dependiente. País que, analizado en estos términos, no podrá jamás pensarse como venido de los barcos, blanco y europeo (Tamagno, 2001).

Los nucleamientos qom de las periferias urbanas dan cuenta, no sólo de las dimensiones económicas, políticas y sociales que atraviesan las ciudades en las cuales se gestan y desarrollan, y los lugares de origen de sus habitantes, sino también, de las formas en que éstos acceden, usan, piensan y significan las tierras ocupadas, transitadas y/o rememoradas, y el modo en que diferentes procesos micro y macro estructurales se entrelazan transformando el campo y la ciudad para dar lugar a nuevas formas de visibilidad indígena. Es en este sentido que las nuevas territorialidades deben comprenderse, tanto en función de las actuales coyunturas como en su historicidad; no siendo, nada más y nada menos, que relaciones de poder que se expresan en el espacio y implican desplazamientos, modificaciones e, incluso, inversiones de las relaciones de fuerza en dicho campo (Maidana, 2008 y Maidana y Tamagno, 2009).

Entendemos que los procesos de territorialización de los qom migrantes son procesos de construcción de territorio - concebido éste en sus aspectos materiales y simbólicos. Hablar de "territorio" implica pensar en términos de una relación entre colectividades y recursos naturales que, necesariamente, pasa por la dimensión política y se refiere a un determinado cuadro jurídico (Pacheco de Oliveira, 2006); implica referenciar un espacio geográfico culturalmente modelado, valorizado y apropiado simbólica e instrumentalmente por una sociedad (Barabas, 2002). Al mismo tiempo, y dado que nos referirnos a territorios de pueblos indígenas, hablamos de "etnoterritorios" para referirnos a aquellos territorios que se establecen en relación con el proceso de identificación étnica y la práctica de la cultura a través del tiempo (Barabas op. cit). Territorios que pueden ser analizados como proyectos en disputa, en tanto las configuraciones sociales y espaciales que han adoptado los qom en el camino de la migración y expresan sus reclamos y sus luchas, así como también son, en parte, respuesta a las políticas públicas con las cuales se articulan las demandas.

El notable aumento en la visibilidad de los pueblos indígenas hace que sus presencias sean notorias, no sólo en los territorios de origen y en la periferia de las ciudades, sino también en las ciudades mismas. Herederos de aquel movimiento poblacional tan bien analizado por Hugo Ratier (1971a, 1971b), reproducen, donde quiera que estén, modos alternos de pensar la vida y habitar los espacios. Habitan la "periferia", pero también transitan el "cen- 
11 Proyecto financiado por el Instituto de Conectividad de las Américas (Canadá), desarrollado por el Instituto Nacional de Antropología y Pensamiento Latinoamericano de la Secretaría de Cultura de la Nación, con el apoyo y participación de otras instituciones, entre ellas la Universidad Nacional de La Plata.

12 Proyecto financiado por el Proyecto de Naciones Unidas para el Desarrollo (PNUD), ejecutado por la Secretaría de Cultura de la Nación e implementado por el Instituto Nacional de Antropología y Pensamiento Latinoamericano (INAPL).

13 Grupos localmente situados formados por capacitadores, promotores y destinatarios.

14 Central obrera, nacida a partir de la separación de un grupo de sindicatos de la Confederación General del Trabajo (CGT) en 1991.

15 Sancionada en el año 2006. tro", se movilizan en busca de empleo para realizar actividades de capacitación, para expresar colectivamente sus demandas en marchas y manifestaciones, a fin de ocupar espacios en las instituciones gubernamentales destinadas a responder a sus demandas y requerimientos.

Durante el año 2006, jóvenes qom de la periferia de la ciudad de La Plata concurrieron, en forma semanal, a la Facultad de Ciencias Naturales y Museo de la Universidad Nacional de La Plata para realizar un "Curso de Capacitación en Tecnologías de Información y la Comunicación para Jóvenes Indígenas”. Esta actividad formó parte de un proyecto más amplio de "Capacitación en Tecnologías de la Información y la Comunicación para Jóvenes Mapuches". ${ }^{11}$ De allí que los cursos de capacitación fueron dictados también en las ciudades de Bariloche, el Maitén, Buenos Aires, Viedma, Esquel y Bahía Blanca, en cada una de las cuales se constituyó un grupo de trabajo o "nodo".

El desarrollo de "Proyecto de Capacitación en Tecnologías de la Información y Comunicación para Jóvenes Toba (Qom)", ${ }^{12}$ durante los años 2008 y 2009, implicó la conformación de tres nodos, ${ }^{13}$ dos en la ciudad de Rosario (Santa Fe), y uno en la ciudad de La Plata (Buenos Aires), donde jóvenes qom concurrieron a los gabinetes informáticos de escuelas locales - en los dos primeros casos - y de la Facultad de Ciencias Naturales y Museo en el último, para realizar la capacitación en forma semanal.

En el año 2007 se reglamentó la Ley 11331 a través del Decreto 3631 que creó el Consejo Provincial de Asuntos Indígenas, máximo organismo de política indígena bonaerense, con participación del Consejo Indígena de la Provincia de Buenos Aires (CIBA) y dependiente de la Secretaría de Derechos Humanos ubicada en el centro de la capital provincial. En el año 2008 se eligieron para el CIBA, dos vocales por pueblo que contara con 3 o más Comunidades en dicha provincia, criterio en base al cual quedarían representados los pueblos Qom, Mapuche y Guaraní.

En el año 2008 al interior de la Central de Trabajadores de la Argentina (CTA) ${ }^{14}$ en el centro de la ciudad de La Plata, la Secretaría de Pueblos Originarios.

El 21 de mayo del 2010, con ocasión de los festejos del Bicentenario, una multitud de gente indígena llegó desde diferentes puntos del país para marchar y congregarse luego en la Plaza de Mayo, reclamando tierra respeto a la diversidad y justicia frente a los atropellos sufridos en el pasado y en el presente.

El 24 de noviembre de 2010, y a pesar de la vigencia de la ley $26160^{15}$ que declara la "emergencia en materia de posesión y propiedad de las tierras que tradicionalmente ocupan las comunidades indígenas originarias del país" (artículo 1º), la policía reprimió ferozmente el corte de ruta que la población qom de La Primavera, provincia de Formosa, realizaba a modo de reclamo por el reconocimiento de la propiedad de la tierra que ocupan. La represión que resultó en el asesinato de Roberto López, dio lugar a un "acampe" que lleva más de 90 días, situado en la intersección de la Avenida 9 de Julio y la Avenida de Mayo, pleno centro de la ciudad de Buenos Aires donde referentes indígenas qom reclaman por justicia y por el esclarecimiento de los hechos, aguardando aún, ser recibidos por las autoridades competentes.

Las presencias de quienes sufrieron las transformaciones abruptas del "medio rural" y migraron llevando consigo el dolor, pero también, una mirada crítica sobre las mismas, cuestiona hoy, no sólo el orden urbano - al desafiar su jerarquía centro-periferia en la cual este último espacio es pensado como el lugar de los pobres -, sino que invalidan el imaginario de ciudad blanca y sin indios. Cuestionan ese mundo urbano que, a pesar de haberse visualizado como el pasaporte a la modernidad, mostró sus limitaciones. Limitaciones que no son más que las limitaciones del desarrollo capitalista y de una sociedad de 
clase fundada en la explotación y la desigualdad. Un orden social que no ha dudado en producir enormes masas de desocupados en función de nuevos modos de acumulación de capital. Los jóvenes de hoy deben ser pensados en estos contextos, sus trayectorias individuales están consignadas por estas historias y sus rebeldías, tienen las improntas de estos procesos, dado que las transformaciones en las subjetividades implican tiempos y profundidades diferentes que las transformaciones en las condiciones materiales de existencia.

\section{CONSIDERACIONES FINALES}

Entender la ciudad como el lugar donde se concentran y expresan las contradicciones, los conflictos y las desigualdades de la sociedad de la cual la ciudad forma parte, posibilita repensar lo urbano y la diversidad, a la luz de los procesos de "territorialización" entendidos como resultados de la articulación entre demandas y políticas públicas.

Reflexionar en términos de procesos de territorialización permite, a su vez, revisar las falencias de interpretar lo urbano como una dimensión esencializada, como un modo de vida substancialmente diferente a lo rural, significa entender lo "rural" y lo "urbano" como construidos en la dinámica sociocultural de la sociedad que los contiene y de la cual son producto. Hoy, el desarrollo tecnológico posibilita la realización de megaemprendimientos vinculados al turismo, a la minería y al agro, que convierten al espacio rural en "presa de la modernidad" otrora fundamentalmente vinculada a los espacios urbanos.

Los modos en que el Chaco de donde provienen los migrantes qom está presente en el cotidiano de los barrios periféricos que habitan, expresado tanto en representaciones como en prácticas, permite señalar que las respuestas dadas por los qom a las condiciones de la vida urbana implican una dialéctica entre la "tradición" y la "modernidad", entre lo "local" y lo "global", entre lo "formal" y lo "informal", entre el presente y el pasado, entre la memoria y los aprendizajes, y por lo tanto, también entre "lo rural" y "lo urbano”. Esta dialéctica nos conduce necesariamente a revisar la comprensión de la dinámica sociocultural de los pueblos indígenas y sus descendientes - donde quiera que estén - en términos de dicotomías.

Así, la presencia de indígenas en las ciudades deja de aparecer como una contradicción o como una paradoja y acaba contribuyendo para una ruptura con el modelo de ciudad europea pensada y diseñada bajo los cánones de la modernidad. La "invisibilidad" que otrora sufrieran los pueblos indígenas, se transforma en emergencia y, la "entelequia" de país venido de los barcos que caracterizó el proyecto de la Generación de 1880, se desvanece para hacernos repensar, no sólo la ciudad, sino la sociedad de la que la misma forma parte, sociedad que no puede dejar de asumir su diversidad.

\section{BIBLIOGRAFIA}

BARABAS, A. Utopias indias: movimientos socioreligiosos en México. México: Grijalbo, 1989 .

. Etnoterritorios y rituales terapéuticos en Oaxaca. Scripta Ethnologica, Buenos Aires, año/Vol.XXIV, n.24, p.9-19, 2002.

BARTOLOMÉ, M. Gente de Costumbre y Gente de Razón. Las identidades étnicas en México. México: INI-Siglo XXI, 1997.
Liliana Ester Tamagno é doutora em Antropología pela Facultad de Ciencias Naturales y Museo (FCNyM) Universidad Nacional de La Plata (UNLP); pesquisadora do Conicet; diretora do Laboratorio de Investigaciones en Antropología Social (LIAS) da Universidad Nacional de La Plata (UNLP). Email: letama5 @yahoo.com.ar

Carolina Andrea Maidana é licenciada em Antropología pela Facultad de Ciencias Naturales y Museo (FCNyM) Universidad Nacional de La Plata (UNLP); bolsista do Conicet; Laboratorio de Investigaciones en Antropología Social (LIAS) da Universidad Nacional de La Plata (UNLP). Email: maidanacarolinaa@ yahoo.com.ar

Artigo recebido em janeiro de 2011 e aprovado para publicação em maio de 2011. 
BARTH, F. The analysis of culture in complex society. Ethnos, Oslo, n.3-4, p.120-42, 1989.

CARDOSO, F.; E. FALETTO. Dependencia y desarrollo en América Latina. México: Siglo XXI, 1969.

CASTELLS, M. La cuestión urbana. Madrid: Siglo XXI, 1979.

CLICHEVSKY, N. Algunas reflexiones sobre informalidad y regularización del suelo urbano. Bitácora 14 (1), Universidad Nacional de Colombia, Bogotá, p.63-88, 2009.

GAMBINI, H. Historia del peronismo. Buenos Aires: Vergara Editor Sociedad Anónima, 2008.

GODELIER, M. Antropologia economica. España: Anagrama, 1978.

HANNERZ, U. Exploring the city. Inquiries toward en urban anthropology. New York: Columbia University Press, 1980.

- Transnational Connections: Culture, People, Places. Londres: Routledge,

1996.

. Fluxos, fronteras, hibridos: palabras clave de la antropología transnacional.

Mana, Río de Janeiro, v.3, n.1, p.7-39, 1997.

IBANEEZ CASELLI, M. A. Sobre los usos de la lengua madre en una situación de bilingüismo en la realidad de un grupo de gente toba en el Barrio de Las Malvinas de la ciudad de La Plata. Actas II Jornadas de Lingüistica Aborigen. Instituto de Lingüística. Buenos Aires: Facultad de Filosofía y Letras - Universidad de Buenos Aires, 1995.

IBAÑEZ CASELLI, M. A.; TAMAGNO, L. Dinámica de la lengua. Diversidad/Homogeneidad. Diferencia/Desigualdad. In: HERZFELD, A.; LASTRA, Y. (Comp.) Causas sociales de la desaparición y del mantenimiento de las lenguas en América Latina. México: Universidad de Sonora, 1999.

LARTIGUE, F. Seminario Internacional Indigenas en el Siglo XXI. Buenos Aires, 1998. LOVE, J. A locomotiva São Paulo na Federação Brasileira 1889-1937. São Paulo: Editora Paz e Terra, 1982.

MAIDANA, C. "Indígenas migrantes urbanos. Destribalización / retribalización - desterritorialización / reterritorialización Actas V Jornadas de Sociología de la UNLP y I Encuentro Latinoamericano de Metodología de las Ciencias Sociales "Cambios y continuidades sociales y políticas en Argentina y la región en las últimas décadas. Desafios para el conocimiento social". Buenos Aires: UNLP, 2008.

MAIDANA, C.; TAMAGNO, L. Migración y lucha por el acceso al suelo urbano. Los qom migrantes. In: BRODA, V. et al. (Coord.) Actas de las V Jornadas Espacio, Memoria e Identidad. Rosario: Centro de Estudios Espacio, Memoria e Identidad (CEEMI) y UNR Editora, 2009.

PACHECO DE OLIVEIRA, J. (Comp.) Hacia una Antropología del Indigenismo. Rio de Janeiro: Contra Capa, 2006.

RATIER, H. Villeros y Villas Miseria. Colección "La historia popular/Vida y milagros de nuestro pueblo", n.60. Buenos Aires: Centro Editor de América Latina, 1971 (Ratier, 1971a).

- El cabecita negra. Colección "La historia popular/Vida y milagros de nuestro pueblo”, n.72. Buenos Aires: Centro Editor de América Latina, 1971 (Ratier, 1971b). TAMAGNO, L. Una comunidad toba en el Gran Buenos Aires: su articulación social. Actas II Congreso Argentino de Antropología Social. Buenos Aires, 1986.

. Indígenas en la ciudad y Políticas Públicas. Lucha por el Acceso a la Vivienda y la Tierra Urbana. 49 Congreso Internacional de Americanistas. Quito, 1997. 
. Nam Qom Hueta à Na Docshi Lmà. Los tobas en la casa del hombre blanco. Identidad memoria y utopía. La Plata: Editorial Al Margen, 2001.

WACQUANT, L. Las cárceles de la miseria. Buenos Aires: Editorial Manantial, 2004.

A B S T R A C T This paper addresses the dialectics tradition/modernity, locallglobal, formallinformal, past /present, memory/learning, related to indigenous migration and the responses of this population to the urban life conditions. The analysis of internal migration movements is understood as the product of individuals or groups looking for jobs and for better living conditions (Castells, 1979 and Tamagno, 2001), and the relationship between urbanization, inequality and diversity is emphasized in what we call "territorialization processes", a concept that allows us to understand how the belonging territories are historically constructed as a result of political, economic and sociocultural factors. these are very relevant issues if we consider the current context of territorial claims and the consequent struggle to legitimize the indigenous presence in urban areas.

K E Y W O R D S City; indians; migration; nucleations; territorialization processes. 